\title{
Exploring stigma and its effect on access to mental health services in unaccompanied refugee children
}

\author{
Pallab Majumder
}

BJPsych Bulletin (2019) 43, 275-281, doi:10.1192/bjb.2019.35

Bulwell Children's Centre, Nottingham, UK

Correspondence to Pallab Majumder (pallab.majumder@nottshc.nhs.uk)

First received 2 Nov 2018, final revision 20 Jan 2019, accepted 27 Mar 2019

(c) The Author 2019. This is an Open Access article, distributed under the terms of the Creative Commons Attribution-NonCommercial-

NoDerivatives licence (http:// creativecommons.org/licenses/by-ncnd/4.0/), which permits non-

commercial re-use, distribution, and reproduction in any medium, provided the original work is unaltered and is properly cited. The written permission of Cambridge University Press must be obtained for commercial re-use or in order to create a derivative work.
Aims and method Despite substantial evidence to show that unaccompanied refugee children suffer a high prevalence of mental illness, their access to services remains poor. One may hypothesise that this is associated with their negative perceptions of mental illness. However, there has been little research exploring this important subject. We aimed to explore unaccompanied refugee children's experiences, perceptions and beliefs of mental illness, focusing on stigma. Fifteen unaccompanied refugee children and 15 carers were interviewed by a semi-structured interview. Thematic analysis was used to analyse data.

Results Three main themes were identified, focusing mainly on issues of stigma related to mental health, mental illness and their treatment, and they were interpreted in detail.

Clinical implications Our findings will contribute to current understanding of stigma and discrimination, and their effect on service engagement, and will hopefully stimulate interest to further explore this area and develop potential solutions.

Declaration of interest None.

Keywords Stigma and discrimination; refugee; refugee children; mental health; mental health service.
Mental illness is seen as a stigmatising or taboo topic in different groups across the population. ${ }^{1}$ Engagement with mental health services may be hindered by people's own perceptions and understanding of mental illness, and stigma and embarrassment can prevent people seeking help. ${ }^{2}$ For the children and young persons who experience mental health difficulties and are engaged with mental health services, there appears to be confusion about the concepts of mental health and mental illness. ${ }^{3}$ Vulnerable young groups, such as those who are in public care, asylum-seeking or homeless, are particularly difficult to engage with mental healthcare services, ${ }^{4}$ adding to their vulnerability to succumb to the poorest mental health outcome. Thus, there is a compelling moral and societal obligation to provide protection to the most vulnerable groups of children and young people in the best possible way. ${ }^{5}$

Currently, more than 51 million people worldwide are displaced from their permanent abode for fear of persecution, torture and death, and this number is increasing. About 33 million have been displaced internally, 17 million have become refugees in adjacent or faraway countries and about 1.2 million are still seeking asylum. This can only be an approximate estimate of the numbers as the situation remains fluid and fragile, with new groups of destitute people adding to the number every day as a result of continual wider geopolitical instabilities. ${ }^{6}$ This has become a global issue, much wider than only the countries or regions involved in the active conflict. About half of the refugee population are minors, and approximately 25000 unaccompanied asylum-seeking children apply for asylum every year across the world.

\section{The triple stigma}

Refugees have the potential to experience an added stigma as a consequence of their status. Many become victims of racial harassment, implying intolerance by some members of the local community. ${ }^{8}$ How they perceive being accepted by the host country also seems to have a significant effect on their psychological functioning. Unaccompanied minors, young refugees who have been separated from both parents and relatives and are not being cared for by an adult, $^{9}$ appear to be at particularly high risk of developing psychological and mental health problems ${ }^{10}$ but are less likely to receive proper psychological care. ${ }^{11}$ Unaccompanied refugee children in contact with mental health services can experience stigma and discrimination related to their asylum seeking or refugee status, having a mental health problem and being unaccompanied or in care - a 'triple stigma'. These facets of stigma are particularly concerning from a cultural perspective. Mental illness can be even more stigmatised in some cultures, which can discourage talking about the subject. ${ }^{12}$ One can speculate that this stigma and resulting discrimination may affect 
mental health service access and eventual outcome of mental well-being. ${ }^{13}$

\section{Understanding the concept of stigma in marginalized groups}

Professionals' understanding of the concepts of mental illness mostly guide mental health service provision. However, public understanding of mental health and illness also has paramount implications. An individual's decision to access treatment can be driven by public perception and attitudes toward people with mental illness. Discrepancies between patients' and clinicians' perceptions may hinder treatment, especially when this originates from different cultural contexts. Haslam proposed four dimensions along which societies can conceptualise mental illness: 'pathologizing' of behaviour as abnormal or deviant; 'moralising', which perceives people with mental illness as accountable for their abnormality; 'medicalising', which views mental health symptoms as having a somatic basis and 'psychologising', which ascribes abnormality to psychological dysfunction that is neither essentialist (like medicalising) nor intentional. ${ }^{14}$ Stress and spiritual concepts of mental illness may also be considered as additional dimensions. Theories of these dimensions, however, do not clarify phenomena such as historical shifts in public perceptions of illness, patterns of service access and help seeking, and discrepancies between lay and professional understandings. Neither do they offer guidance on possible ways to improve service engagement, outcome and mitigate stigma.

The issue of stigma, often resulting in discrimination, attached to marginalised groups such as people with mental illness and refugees can perhaps be better understood by exploring the experiences, perceptions and beliefs of unaccompanied refugee children. This is because, in their lived experiences, there exist a number of different dimensions of being stigmatised and therefore discriminated against, as mentioned above. This will also allow us to develop a better understanding of the possible ways to mitigate the damaging effects of these phenomena, and thereby facilitate the process of better engagement and integration of these marginalised groups with services. There is an inadequate amount of qualitative research with unaccompanied refugee children addressing the topics of mental illness and stigma at present, and thus requires greater attention to help us develop a more comprehensive understanding of these pertinent issues. Therefore, the aim of this paper is to explore the beliefs and perceptions of unaccompanied refugee children in the context of mental illness, its treatment, service engagement and the stigma attached to it. Another objective is to develop a better understanding of the possible mitigating factors that can potentially be applied to other marginalised groups to facilitate their engagement with services.

\section{Method}

The main research project from which this paper is developed explored the general perceptions and beliefs of unaccompanied refugee children with regards to their experience of the mental health services. However, this paper focuses on the subject of stigma attached to the concept of mental illness; its effect on these children's service access, utilisation and engagement; and possible ways to tackle the issue of stigma to improve service engagement.

\section{Sample}

The sample was recruited mainly from consecutive referrals of unaccompanied refugee children to the specialist mental

\begin{tabular}{|c|c|c|c|c|}
\hline Number & Gender & Age in years & Nature of the mental health problem & Country of origin \\
\hline 1 & M & 18 & Depression, self-harm & Afghanistan \\
\hline 2 & M & 15 & PTSD, anxiety & Iran \\
\hline 3 & M & 17 & PTSD, depression, anxiety & Afghanistan \\
\hline 4 & M & 17 & Self-harm, adjustment reaction & Afghanistan \\
\hline 5 (Declined) & $\mathrm{F}$ & 18 & PTSD, substance misuse & Somalia \\
\hline 6 & $\mathrm{~F}$ & 17 & PTSD, depression, self-harm & Eritrea \\
\hline 7 & M & 15 & Intellectual disability, dissociative disorder, PTSD & Afghanistan \\
\hline $8^{a}$ & M & 17 & Psychosis & Somalia \\
\hline 9 & M & 16 & Self-harm, PTSD, anxiety & Afghanistan \\
\hline 10 & M & 17 & PTSD, depression & Afghanistan \\
\hline 11 & M & 16 & Self-harm, adjustment reaction & Iran \\
\hline 12 & M & 17 & Self-harm & Afghanistan \\
\hline 13 & M & 18 & Depression, adjustment reaction & Afghanistan \\
\hline 14 & M & 15 & Depression, self-harm, substance misuse & Afghanistan \\
\hline 15 & M & 18 & PTSD, self-harm & Afghanistan \\
\hline 16 & M & 18 & PTSD, impaired sleep & Afghanistan \\
\hline
\end{tabular}

PTSD, post-traumatic stress disorder. 


\begin{tabular}{|c|c|c|c|c|}
\hline Number & Nature of carer & Gender & Nature of mental health problem of the child & Child's country of origin \\
\hline 1 & Foster & Female & Depression, self-harm & Afghanistan \\
\hline 2 & Foster & Female & PTSD, anxiety & Iran \\
\hline 3 & Foster & Female & PTSD, depression, anxiety & Afghanistan \\
\hline 4 & Foster & Female & Self-harm, adjustment reaction & Afghanistan \\
\hline 5 & Residential & Female & PTSD, substance misuse & Somalia \\
\hline 6 (Declined) & Foster & Female & PTSD, depression, self-harm & Eritrea \\
\hline 7 & Residential & Male & Intellectual disability, dissociative disorder, PTSD & Afghanistan \\
\hline 8 & Social worker & Male & Psychosis & Somalia \\
\hline 9 & Foster & Female & Self-harm, PTSD, anxiety & Afghanistan \\
\hline 10 & Foster & Male & PTSD, depression & Afghanistan \\
\hline 11 & Foster & Male and female & Self-harm, adjustment reaction & Iran \\
\hline 12 & Foster & Male and female & Self-harm & Afghanistan \\
\hline 13 & Residential & Female & Depression, adjustment reaction & Afghanistan \\
\hline 14 & Residential & Male & Depression, self-harm, substance misuse & Afghanistan \\
\hline 15 & Foster & Female & PTSD, self-harm & Afghanistan \\
\hline 16 & Foster & Female & PTSD, impaired sleep & Afghanistan \\
\hline
\end{tabular}

PTSD, post-traumatic stress disorder.

health service. Participation was requested by contacting the participants through social workers from two separate local authorities in the central part of UK. A total of 15 young persons were recruited to the study (Table 1). They were between 15 and 18 years of age, mostly male and typically from either Arab or East African countries. The carers of these young persons were also invited to take part in the study (Table 2).

\section{Data}

Informed consent was obtained from the young person and the person with parental responsibility (the social worker) for the unaccompanied refugee child to participate, and from the carer for the carer to participate. Data was then collected through the process of a detailed semi-structured interviewing. Semi-structured interviewing is a useful technique that can be used flexibly to explore abstract concepts such as stigma and discrimination. To minimise the likelihood of any important theme being missed, data collection through interviews was continued until a thematic saturation had been achieved. ${ }^{15}$ This was done by carrying on collecting data until no new themes were emerging consistent with Francis et al's ${ }^{16}$ guidelines on qualitative interviewing. The interviews were audio-taped and notes were taken and later transcribed verbatim for detailed analysis.

\section{Analysis}

At the end of the data collection phase, there were 30 transcripts in total. Data was analysed by thematic analysis. This helps identify certain patterns in the data corpus that eventually emerges into the shape of 'themes'. Thematic analysis was chosen as it is very useful in drawing underlying meanings from an apparently unrelated data-set and has the necessary theoretical flexibility to be utilised in analysing most qualitative data. ${ }^{17}$ The data from the transcriptions was analysed by two different researchers independently to ensure reliability, and the emerged themes are loyal to the original data collected from the participants. The patterns elicited were subsequently organised into themes. These, in turn, were systematically analysed and synthesised to attempt to answer the research aims, as well as to develop any potential hypotheses adding to our knowledge base in this area.

\section{Ethics}

To ensure that the participation of this group of vulnerable and marginalised young persons remains ethically sound, all possible general (e.g. acquiring informed consent from a child) and specific (e.g. chances of implicit coercion in an asylum-seeking mentally ill individual who is being looked after by the state) sensitive ethical issues were rigorously considered. As a result, ethical approval was finally obtained from the Regional Ethics Committee of the National Research Ethics Service (UK).

\section{Results}

After analysing the transcripts from in-depth interviews with young persons and their carers, focusing on stigma, three main themes emerged. In the first theme, I explore the negative perceptions of mental illness in this population, and in the second, the possible reasons for such perceptions. In the final theme, I examine the young persons' resistance in accepting their own mental health difficulties and its possible underlying mechanisms. 


\section{Theme 1: negative perceptions of the concept of mental illness}

Fourteen out of fifteen young people and many carers expressed their opinions on the young person's negative perceptions of mental illness. Contact with mental health services in the UK did not appear to shift such perceptions. They referred to mental illness by terms like 'crazy', 'mental' or 'mad', interchangeably. This is again consistent with findings from other young vulnerable groups. ${ }^{1}$ The narrative of mental illness described by several young persons had a striking similarity, in associating a mentally ill person with someone who has lost all sense of basic upkeep, hygiene, dressing and hair; is locked up in a hospital or prison; sleeps on the streets and drinks alcohol; and is being beaten up or stoned.

'...Then I told this lady I'm not crazy, I'm not like these, these, you know...I tell her look my hair, look my clothes, I'm not crazy.' Young person 15.

'...They just don't know what they're doing. Some of them been locked in the hospital, or in the prison. I seen lots of mentals in my country.' Young person 1.

'The mental is like people like, you know, crazy or mad and their mind doesn't work, and some people drink a lot, they go in mental hospital.' Young person 13.

Yeah, the idea of mental health is, you know, over there is somebody's mad. He was telling me that we can stone him and beat him...' Carer 9.

The carers, on the other hand, seemed insightful into these young persons' beliefs, as they considered the sources of their negative perceptions of mental illness. They reflected that the society and culture possessed a high level of stigma and negative views of mental illness, which discouraged acceptance and admission by those who suffered problems.

'I might be making a bold statement here, but mental health with black and Asian minority ethnic communities is a taboo anyway. People don't say it...' Carer 8.

$\therefore$ If people had them, then it would be, almost frowned on or discouraged in his culture from saying I've got these kind of problems.' Carer 11.

‘..In some cultures, mental health is not perceived the way that we perceive it in the UK, or America or the Western world. You know, some cultures would just say that you're a crazy person perhaps, in terms of summing it up. And therefore the stigma associated with that would consequently lead people not to admit it.' Carer 12 .

'Thinking about it, all of the asylum seekers, there are now eight of them, they needed this help but to them, all of them, it was the same, same answer from them, so it must be something from back home. The perception about this, anything to do with the term "mental health" was the same.' Carer 16.

This may have influenced the young persons' views on mental illness, and their reluctance to admit their own mental health difficulties. One of the abovementioned carers stated that he 'would be almost frowned on or discouraged in his culture from saying I've got these kind of problems'. This has clear implications for service engagement.

It can be suggested from the above accounts that such attitudes and perceptions of mental illness would affect these young people's engagement with treatment. However, at this stage, this inference still remains a hypothesis that requires further substantiating. Some carers came up with suggestions such as avoiding the terms 'mental illness' or 'mental health' to describe the service, instead replacing them with more neutral words.

'It's a shame unless you know, instead of saying "mental health" it can be changed into something else, right? Just the word, not mental health issues.' Carer 16.

‘. . Say it's [CAMHS] not called mental or medical terms, it's just called a holiday camp or something nice, a name which has got some nice name like, you know, Butlins has got a nice name.' Carer 5.

$\therefore$...He says he's not mental, why would he want to go and see a mental health service... if they could just remove that "mental health" and use it as "Westcotes House" (mental health service building)... if they have different headings for services then, I don't know, you might find you get better results.' Carer 14.

Participants' statements revealed that the young persons possessed a negative view of mental illness and anybody suffering from it. This is evident in the terms used by the young persons to describe mental illness as well as in their narratives of someone suffering from these difficulties. It is hypothesised that this can at least partially be attributed to the stigma attached to mental illness in the young persons' countries of origin, which also influences their behaviour with regard to acknowledging such problems and necessary interventions.

\section{Theme 2: anticipated social implications of suffering from mental illness}

Some of the young persons expressed their worries about the anticipated consequences of being mentally ill. This was corroborated by their carers. Many participants, both young persons and carers, talked about the young person's anxiety that they might eventually be incarcerated in a secure mental hospital, asylum or prison. Again, there seems to be an important cultural relevance, as the young person's views are likely to be intrinsically tied to what tends to happen in their own countries of origin.

'Some of them been locked in the hospital, or in the prison. 1 seen lots of mentals in my country...So sometime I just think if you grow up or just get more worse, you're going to become one of them.' Young person 1.

'When you disabled, you go in disabled house.' Young person 15.

'He was always thinking that, you know, he might end up in a mental hospital.' Carer 9.

'...For them it was mad, they are mad. So they should be put in mad asylums.' Carer 16.

Worries appeared to be quite deep-seated across the whole sample. Young persons' and carers' responses reflected their fears of becoming socially isolated as a result of their mental illness. One participant commented that mental illness would lead to sleeping rough on the streets.

II don't know about mental...Sleep on street and go crazy, innit.' Young person 4.

As per other young persons' and carers' accounts, social isolation can actually be due to abandonment by their friends and family. The prospect of social isolation was supported by a carer's statement about the young person in her care that he would also lose his friends if they came to know 
about his mental illness or that he received help from mental health services.

'... So sometime my friends don't wants to be with me because I've got this problem.' Young person 1.

'Going there [to CAMHS] for his mental assessment to him it was, no, none of my friends went...they won't be my friends once they come to know. I said they don't need to know, he said no, but, they'll know.' Carer 16.

Reflecting on the same subject of anticipated isolation and abandonment by society, carers considered some of the young persons' culture of origin, where mental health can possibly lead to not being taken care of properly and difficulty in getting married.

‘. .In those cultures he would have it as somebody who is mad, is mad, you know, he's mad. Nobody would look after him properly, you know, and see what's wrong like we do it here.' Carer 9.

'Because, um, in the Somalian culture...say from a female's point of view, say if there is a father and mother and their young female daughter wanted help, because then the word gets round it's difficult for them then to get them married off and it affects their future.' Carer 5.

Young persons' anxiety of the consequences of suffering from mental illness is apparent in their statements. These include being incarcerated and institutionalised, or suffering a downward social drift and social isolation. These beliefs may have far-reaching implications on their views of mental health services and professionals, and ultimately on helpseeking for their own mental health difficulties.

\section{Theme 3: denial of mental illness}

Throughout this research, for the purpose of consistency, young people who were being treated by the specialist mental health service were described as having presented with 'mental illness'. However, the Western cultural notion of mental illness may be substantially different from what applies to other cultures. ${ }^{18}$ There is, therefore, a risk of approaching their predicament through a Western professional 'goldfish bowl' view ${ }^{19}$ of mental illnesses by interpreting their experiences as diagnostic categories of Western systems of psychiatric classification. ${ }^{20}$ Many young persons denied having any mental illness despite having attended a mental health service. This seems to be linked to the earlier themes of social stigma and fears of untoward social consequences. They appeared to be embarrassed about discussing their own mental health difficulties, which was expressed by an increasingly hesitant speech; by evading or altogether avoiding using terms such as 'mental health'; or by giving alternative explanations for seeing a psychiatrist, such as physical health problems.

'Um, first of all, I don't have any, I don't know, uh, I mean, I'm not, um, mental problem. I got, I saw the bad dream, I didn't sleep then, sometimes. Uh, maybe that thing, I don't know, maybe, that's why...'Young person 6 .

Interviewer: 'So when you asked about mental health, he avoided the term.'

Interpreter: 'That term...Other things except that.' Young person 7 .

Interpreter: 'He is saying that he's going to $P$ [Psychiatrist] because he's got a problem with his eyes, he's got a headache...' Young person 7.
Both young persons 6 and 7, despite having significant contacts with child and adolescent mental health services, showed a tendency to deny their mental health difficulties. Carers also agreed with this notion of the young persons' propensity to avoid talking about or denying their mental health problems.

'It's sensitive for some young people to admit that they've been to CAMHS and that they need that sort of help.' Carer 13.

'They don't see themselves as mentally unwell because when they think of crazy, they think of people who have really, really big problems.' Carer 15.

The reasons that the carers attributed to this denial included young persons' 'sensitivity' to mental illness. This again points toward the social and cultural stigma attached to the concept of mental illness. Carers also reaffirmed the notion that mental illness for refugee minors can mean only having a severe mental disorder, which may have influenced their engagement with service and explain at least part of the stigma attached to their construction.

\section{Discussion}

Refugee young persons' perceptions of mental illness seem to be predominantly influenced by their sociocultural construction of such concepts. The idea of 'madness' as the most prevalent understanding of mental health difficulties, the feared consequences of social isolation in the forms of abandonment and rejection by friends and family, and the eventual downward drift of social and personal life can all explain young persons' reluctance to accept their mental health difficulties and to engage with mental health services.

Refugee young persons' perceptions of mental health and mental illness, and the associated social stigma, have important implications for their engagement with professionals, services and eventually treatment outcome, as well as efficacy of the service provided. In this research, the majority of the young persons' understanding of mental illness was affected by their negative views of the construct. Previous researchers repeatedly identified the reluctance of this group to access mental health services and to engage in treatment. Stigma associated with mental illness and treatment in their countries of origin was depicted as a major underlying reason. ${ }^{13,21-23}$ In the present study, some young persons preferred to express their difficulties through physical or somatic symptoms. This might be, again, a reflection of the social stigma attached to the construct of mental illness. We should note further that in many cultures, conceptions of mental health difficulties are very different from biomedical concepts common in the Western sociocultural framework. In some cultures, psychological problems are understood by referring to physical symptoms and in many languages, terms equivalent to English concepts do not exist. Honwana ${ }^{18}$ commented that the social and cultural notions of physical health, mental health and healing that apply to these children are often very different from the Eurocentric view, and that addressing such culture-specific perceptions may be fundamental in improving their wellbeing. Negotiation between the health beliefs of refugees 
and those of their host country could be an important step in mitigating this effect. ${ }^{24}$

The implications of suffering from mental illness in a particular sociocultural context may influence the stigma attached to those conditions. It is important to note that until recently, at least in certain parts of the world, significant social stigma was associated with physical illnesses like leprosy and tuberculosis, and is still associated with HIV. ${ }^{25,26}$ The negative effect of social stigma associated with these physical illnesses perhaps started to reduce only after the paradigm shift in our understanding of the pathophysiological processes and development of effective preventative and treatment measures. ${ }^{25}$ Subsequently, scientific explanation of these conditions could become the predominant social and public narrative to replace inaccurate and distorted beliefs. On the other hand, there remain serious issues surrounding investment of resource and prioritising development of evidence-based adequate mental health service framework in most of the countries of origin of these young persons. ${ }^{27,28}$ As a result, these children's perception of mental illness and intervention remains mostly based on the available alternative narratives, often heavily influenced by stigma and misconceptions.

Attempting to draw a parallel, in the West, both in clinical practice and public discourse, language seems to be an important factor influencing the stigma surrounding mental illness. Socially acceptable expressions have been developed and changed repeatedly over time to describe mental illness and related conditions. Scientific terminologies used to describe developmental delay, for example, moved from 'imbecile', 'moron' or 'idiot', to 'mental retardation', 'learning disability', 'special need' and eventually 'intellectual disability' terms to describe serious mental illnesses shifted from 'lunatic' or 'insane' to 'psychosis'. Since the older language used to describe any mental illness or learning impairment shows a propensity to be associated with stigma, a constant need is perceived by society to develop newer and less stigmatising language. This, however, appears to be a symptomatic intervention in the absence of a definitive scientific understanding and treatment for many of these conditions. The direction of the association between language and stigma may therefore be that, the stigma originating from the fear of the society of the 'unknowns' of mental illness influences the constant periodic shift in the language used to describe mental illness, rather than language influencing stigma. Interestingly, fear of unknown may also be argued as an important underlying factor responsible for stigmatisation and discrimination against refugees and other marginalised groups of population in the society. ${ }^{29}$

Although the search for a more accurate scientific explanation for many mental illnesses and their treatment goes on, what can the practitioners, researchers and policy makers in this field do to tackle the stigma attached to mental illness and its treatments in a more effective and sustained manner? Summerfield ${ }^{30}$ questioned the extent to which the pain, suffering and difficult experiences of refugees can be reduced to a matter of mental health. This brings home the notion of whether it is important now, more than ever, to shift the narrative of service provision toward 'wellbeing' rather than illness. The purpose of asking this question is to open up a discussion, rather than leaning toward either side of the debate, on how we should describe our services to the public that would maximise the access of difficult-to-engage populations and minimise the effects of perceived social stigma.

\section{Limitations}

There are several limitations to this study. The researcher's subjectivity and social construction may have influenced the interpretation of data, as in any other qualitative analysis. The participating young persons were predominantly male Afghan nationals (Table 1), with only one female participant (from Eritrea). These demographic factors, along with the fact that this is a qualitative study that aims to explore, interpret and hypothesise on the basis of the current sample only, limits the generalisability of the study findings. There were a number of challenges in recruiting, obtaining data and conducting research within this hard-to-reach population. Examples of these are the occasional fragmented nature of their narratives, requirement of interpreters and convincing the gatekeepers to agree for the young persons to be contacted. However, eventually the participants were successfully engaged in the process and were able to narrate their experiences effectively. There is also a possibility of social desirability bias driven by the participants' apprehension about possible implications of admitting psychological symptoms. The power difference in a situation where an unaccompanied young person being interviewed by a professional in a foreign country in which they are seeking asylum, could affect the interviewees' openness. Attempts were made to mitigate this effect by researchers not being involved in the clinical care of the participants, and explaining at the outset, the independent and distant position of the interviewers from any other agencies and professionals. Despite these measures, the potential influence of the power difference could have persisted. Future research may be carried out using quantitative or mixed methods, longitudinal studies with larger and more heterogeneous demographic variables to test the generalisability of these findings, and elicit possible factors associated with mitigation of these obstacles.

\section{About the author}

Pallab Majumder, MBBS, MRCPsych, MD, PhD, is an Honorary (Consultant) Assistant Professor at the University of Nottingham, and a Consultant Child and Adolescent Psychiatrist at Nottinghamshire Healthcare NHS Foundation Trust, UK.

\section{Acknowledgements}

I would like to thank the participating young people, their carers who consented to participate in this study, and social workers and clinical professionals who made access possible without whom this project would not have been completed.

\section{References}

1 O' Reilly M, Taylor H, Vostanis P. 'Nuts, schiz, psycho': an exploration of young homeless people's perceptions and dilemmas of defining mental health. Soc Sci Med 2009; 68: 1737-44. 
2 Royal College of Psychiatrists. Attitudes Towards Depression. Royal College of Psychiatrists, 1995.

3 O'Reilly M. 'What seems to be the problem?' A myriad of terms for mental health and behavioural concerns. Disabil Stud Q 2005; 25: 4.

4 Vostanis P (ed). Mental Health Interventions and Services for Vulnerable Children and Young People. Jessica Kingsley Publishers, 2007.

5 Grodin MA, Glantz LH. Children as Research Subjects, Science, Ethics and Law. Oxford University Press, 1994

6 United Nations High Commissioner for Refugees. Mid-Year Trends 2014. United Nations High Commissioner for Refugees, 2015 (http://www unhcr.org/dach/wp-content/uploads/sites/27/2017/04/Mid-Year Trends_2014.pdf).

7 United Nations High Commissioner for Refugees. Global Trends 2013. United Nations High Commissioner for Refugees, 2014 (http://www. unhcr.org/5399a14f9.pdf).

8 Rutter J, Cooley L, Reynolds S, Sheldon R. From Refugee to Citizen: 'Standing on my Own Two Feet' A Research Report On Integration, 'Britishness' and Citizenship. Metropolitan Support Trust and the Institute of Public Policy Research, 2007 (https://www.metropolitan. org.uk/images/From-Refugee-to-Citizen-Exec.pdf).

9 United Nations High Commissioner for Refugees. United Nations Convention of the Rights of the Child. United Nations High Commissioner for Refugees, 1989 (http://www.unhcr.org/cgi-bin/texis/vtx/home/ opendocPDFViewer.html?docid=50f941fe9\&query=rights).

10 Fazel M, Wheeler J, Danesh J. Prevalence of serious mental disorder in 7000 refugees resettled in western countries: a systematic review. Lancet 2005; 365: 1309-14.

11 Ellis $\mathrm{BH}$, Miller $\mathrm{A}$, Baldwin $\mathrm{H}$, Abdi S. New directions in refugee youth mental health services: overcoming barriers to engagement. J Child Adolesc Trauma 2011; 4: 69-85.

12 Delago M, Jones K, Rohani M. Social Work Practice With Refugee and Immigrant Youth. Pearson, 2005.

13 Lustig SL, Kia-Keating M, Knight WG, Geltman P, Ellis H, Kinzie JD, et al. Review of child and adolescent refugee mental health. J Am Acad Child Adolesc Psychiatry 2004; 43: 24-36

14 Haslam N. Dimensions of folk psychiatry. Rev Gen Psychol 2005; 9: 35-47.

15 O'Reilly M, Parker N. Unsatisfactory saturation: a critical exploration of the notion of saturated sample sizes in qualitative research. Qual Res 2013; 13: 190-7.

16 Francis J, Johnston M, Robertson C, Glidewell L, Entwistle V, Eccles MP, et al. What is an adequate sample size? operationalizing data saturation for theory-based interview studies. Psychol Health 2010; 25: 1229-45.
17 Braun V, Clarke V. Using thematic analysis in psychology. Qual Res Psychol 2006; 3: 77-101.

18 Honwana A. Chapter 13: Child soldiers: community healing and rituals in Mozambique and Angola. In International Perspectives on Youth Conflict and Development: 225-44. Oxford University Press, 2006.

19 Thomas S, Thomas S, Nafees B, Bhugra D. 'I was running away from death': The pre-flight experiences of unaccompanied asylum seeking children in the UK. Child Care Health Dev 2004; 30: 113-22.

20 Majumder P. Service development in the new NHS. Br J Healthc Manag 2014; 20: 224-9.

21 Griffith P, Chan-Kam S. Chapter 11: why do we hate refugees? Public opinion, citizenship and integration. In Reclaiming Britishness (eds $\mathrm{P}$ Griffith, M Leonard): 91-103. London Foreign Policy Centre, 2002.

22 De Anstiss H, Ziaian T. Mental health help-seeking and refugee adolescents: qualitative findings from a mixed-methods investigation. Aust Psychol 2010; 45: 29-37.

23 Michelson D, Sclare I. Psychological needs, service utilization and provision of care in a specialist mental health clinic for young refugees: a comparative study. Clin Child Psychol Psychiatry 2009; 14: 273-96.

24 Palinkas LA, Pickwell SM, Brandstein K, Clark TJ, Hill LL, et al. The journey to wellness: stages of refugee health promotion and disease prevention. J Immigr Health 2003; 5: 19-28.

25 Nsagha DS, Bissek A-CZK, Nsagha SM, Njunda AL, Assob JCN, et al. Social stigma as an epidemiological determinant for leprosy elimination in Cameroon. J Public Health Afr 2011; 2: e10.

26 Chambers LA, Rueda S, Baker DN, Wilson MG, Deutsch R, et al. Stigma, HIV and health: a qualitative synthesis. BMC Public Health 2015; 15: 848.

27 Vostanis P. Editorial: global child mental health - emerging challenges and opportunities. Child Adolesc Ment Health 2017; 22: 177-8.

28 Getanda EM, Vostanis P, O'Reilly M. Exploring the challenges of meeting child mental health needs through community engagement in Kenya. Child Adolesc Ment Health 2017; 22: 201-8.

29 Winters J. Why We Fear the Unknown. Psychol Today, 2002 (https://www. psychologytoday.com/articles/200205/why-we-fear-the-unknown).

30 Summerfield D. Childhood, war, refugeedom and 'trauma': Three core questions for mental health professionals. Transcult Psychiatry 2000; 37: 417-33

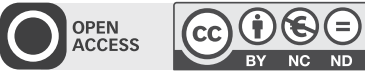

